\title{
UNIQUE INTEGRABILITY OF CONTINUOUS $k$-PLANE FIELDS
}

\author{
PATRICK D. MCSWIGGEN
}

(Communicated by Linda Keen)

\begin{abstract}
It is shown that unique integrability of a continuous $k$-plane field is a generic property for plane field satisfying a "topological bracket condition".
\end{abstract}

This note was motivated by the general observation that in a dynamical system it is quite common for there to occur an invariant plane field which is only continuous, but which nevertheless integrates uniquely. (For example, the stable and unstable planes of an Anosov diffeomorphism). For any given example there are, of course, more immediate explanations of why the integral manifolds should be unique. However, it seems surprising considered just in terms of the general problem of integrating continuous plane fields. In this note we show that a result on the generic integrability of continuous vector fields in [4] (see [2, p. 121]) can be extended to plane fields, and this shows that, in general, unique integrability of continuous plane fields is not uncommon.

Let $M$ be a not necessarily compact manifold of dimension $n$. ( $M$ could, for example, be an open ball.) Let $\mathscr{X}_{k}^{r}(M), 0<k<n$, be the space of $C^{r}$ $k$-plane fields on $M$, where $M$ is assumed to be a manifold that admits such plane fields. That is, $\mathscr{X}_{k}^{r}(M)$ is the space of $C^{r}$ sections of the Grassmann bundle, $G_{k}(M)$, of $k$-planes tangent to $M$. As a function space we assume $\mathscr{X}_{k}^{r}(M)$ has been endowed with either the weak or the strong topology [3]. For $r=0$, the weak topology on $\mathscr{Z}_{k}^{0}(M)$ is the topology of uniform convergence on compact sets.

If $E \in \mathscr{Z}_{k}^{r}(M)$, then $E$ is a $C^{r}$ subbundle of $T M$ of dimension $k . E$ is integrable if for every $x \in M$ there is a (local) $C^{r+1}$ submanifold $N \subset M$ through $x$ whose tangent bundle is $E$ restricted to $N$. We say that a $C^{1}$ plane field $E$ is involutive if for any two $C^{1}$ vector fields $X$ and $Y$ taking values in $E,[X, Y]$ again takes values in $E$. For $C^{0}$ plane fields we make the following definition.

Definition. We say $E \in \mathscr{Z}_{k}^{0}(M)$ is topologically involutive if $E$ is the weak limit of smooth (at least $C^{1}$ ) involutive plane fields.

Frobenius' Theorem [1] states that for $r \geq 1, E$ is integrable if and only if it is involutive, in which case it is uniquely integrable. For continuous plane fields,

Received by the editors October 18, 1993.

1991 Mathematics Subject Classification. Primary 58A30; Secondary 58F18. 
not all integrable plane fields need to be topologically involutive. However, we do have the following.

Proposition. If $E \in \mathscr{X}_{k}^{0}(M)$ is topologically involutive, then $E$ is integrable.

Proof. Let $x \in M$. If $E$ is topologically involutive, then there exist smooth involutive plane fields $E_{i} \rightarrow E$ uniformly near $x$. Let $N_{i}$ be the (local) integral manifold of $E_{i}$ through $x$, and let $V_{x}$ be a plane in $T_{x} M$ complementary to $E_{x}$. Since $E$ is continuous, there exists a neighborhood $U$ of zero in $E_{x}$ such that for each $i$ (in exponential coordinates), $N_{i}$ over $U$ is the graph of a $C^{1}$ function $g_{i}: U \rightarrow V_{x}$. The family $\left\{g_{i}\right\}$ is equicontinuous and bounded, and hence precompact. Therefore, there exists a subsequence converging to some $C^{1}$ function $g: U \rightarrow V_{x}$. The graph of $g$ is then an integral manifold of $E$ through $x$.

By definition, the subset of $\mathscr{X}_{k}^{0}(M)$ of topologically involutive plane fields is weakly closed. A weakly closed subset in either the weak or strong topology is a Baire space [3]. It is well known that a continuous plane field which is integrable need not be uniquely integrable. However, the following shows that unique integrability is a generic property of topologically involutive plane fields.

Theorem. The set of uniquely integrable continuous $k$-plane fields on $M$ is residual in the topologically involutive $k$-plane fields.

Proof. Let $\left\{K_{i}\right\}$ be a countable cover of $M$ by compact sets, and let $\mathscr{I}\left(K_{i}\right)$ be the set of topologically involutive plane fields on $M$ with the following property. For any $E \in \mathscr{I}\left(K_{i}\right)$, if $N_{1}$ and $N_{2}$ are any local integral manifolds of $E$ through a point $x \in K_{i}$, then $N_{1}$ and $N_{2}$ agree on a neighborhood of $x$. Clearly, $\bigcap_{i} \mathscr{I}\left(K_{i}\right)$ is the set of uniquely integrable plane fields. Therefore, it will be sufficient to show that $\mathscr{I}(K)$ is residual for any compact $K \subset M$.

A set is residual if and only if it is residual relative to each element of some open cover. That is, its intersection with each element is residual in that element. Therefore, it will be sufficient to show $\mathscr{I}(K)$ is residual relative to some neighborhood of any fixed, arbitrary topologically involutive plane field $E$.

Fix a topologically involutive plane field $E$. For $x \in K$, choose local coordinates on a neighborhood $U$ of $x$ identifying $x$ with the origin and $E_{x}$ with $\mathbb{R}^{k} \times\{0\}$. By identifying an element $\mathbf{A} \in L\left(\mathbb{R}^{k}, \mathbb{R}^{n-k}\right)$ with the plane $\operatorname{graph}(\mathbf{A}):=\{(v, \mathbf{A} v)\} \in G_{k}(M)$ in these local coordinates, we can define a neighborhood $W$ of $E_{x}$ in $G_{k}(M)$ by $W=U \times\{\mathbf{A}:\|\mathbf{A}\|<1\}$. $E$ is continuous, so if $U$ is sufficiently small, $E(U) \subset W$. Let $D_{r}^{m}$ denote the closed disk of radius $r$ in $\mathbb{R}^{m}$, and choose $d>0$ such that $B:=D_{2 d}^{k} \times D_{2 d}^{n-k} \subset U$. Let $C:=D_{d}^{k} \times D_{d}^{n-k}$.

Choose a finite collection $\left\{\left(U_{i}, W_{i}, B_{i}, C_{i}\right)\right\}$ as above such that $\cup C_{i} \supset K$, and define a neighborhood $\mathscr{N}$ of $E$ by $\mathscr{N}=\left\{F: \forall i F\left(B_{i}\right) \subset W_{i}\right\}$. We want to show $\mathscr{I}(K)$ is residual relative to $\mathscr{N}$. Let $F \in \mathscr{N}, x \in C_{i}$ for some $i$, and let $N_{1}$ and $N_{2}$ be any two local integral manifolds of $F$ through $x$. Write $x$ in the local coordinates on $U_{i}$ as $\left(u_{0}, v_{0}\right) \in D_{d}^{k} \times D_{d}^{n-k}$. By our choice of $\mathscr{N}, N_{1} \cap$ int $B_{i}$ is the graph of a $C^{1}$ function $g_{1}: V_{1} \rightarrow \mathbb{R}^{n-k}$, where $V_{1} \subset D_{2 d}^{k}$ is the projection (in local coordinates) of $N_{1}$ into $\mathbb{R}^{k}$, and is some neighborhood of $u_{0}$. Likewise, $N_{2}$ is the graph of some $g_{2}: V_{2} \rightarrow \mathbb{R}^{n-k}$. Set $\hat{r}=\sup \left\{r \leq d\right.$ : disk centered on $u_{0}$ with radius $\left.r \subset V_{1} \cap V_{2}\right\}$. Let 
$\delta_{i}\left[N_{1}, N_{2}\right]:=\sup \left\{\left\|\left(g_{1}-g_{2}\right)(u)\right\| /\left\|u-u_{0}\right\|:\left\|u-u_{0}\right\| \leq 1 / 2 \hat{r}\right\}$. This is finite since $\left(g_{1}-g_{2}\right)$ is $C^{1}$, and $N_{1}, N_{2}$ both contain $x \Rightarrow g_{1}\left(u_{0}\right)=v_{0}=g_{2}\left(u_{0}\right)$. $\delta_{i}\left[N_{1}, N_{2}\right]$ measures the rate at which $N_{1}$ and $N_{2}$ diverge (as measured by their rate of vertical separation in local coordinates, but only over a disk which is common to both). Note that $\delta_{i}\left[N_{1}, N_{2}\right]$ is well defined for any two integral manifold of $F$ through $x \in C_{i}$.

For $\rho>0$ define a set $\mathscr{I}_{\rho} \subset \mathcal{N}$ as follows. $F \in \mathscr{I}_{\rho}$ iff for any $i$, any $x \in C_{i}$, and any integral manifolds $N_{1}, N_{2}$ of $F$ through $x, \delta_{i}\left[N_{1}, N_{2}\right]<$ $\rho$. Suppose $F \in \bigcap_{n \geq 1} \mathscr{J}_{1 / n}$, and let $N_{1}$ and $N_{2}$ be a fixed pair of integral manifolds of $F$ through $x \in K$. Then $x \in C_{i}$ for some $i$, and so $\delta_{i}\left[N_{1}, N_{2}\right]<$ $1 / n$ for all $n$. Hence, $g_{1}(u)=g_{2}(u)$ for all $u$ with $\left\|u-u_{0}\right\| \leq 1 / 2 \hat{r}$, and $\hat{r}$ depends only on the overlap $V_{1} \cap V_{2}$. Therefore, $N_{1}$ and $N_{2}$ agree on a neighborhood of $x$. Since this holds for any $x \in K$, we have $\bigcap_{n>1} \mathscr{J}_{1 / n} \subset$ $\mathscr{F}(K)$. We claim that for any $\rho>0, \mathscr{I}_{\rho}$ contains an open and dense set, which will complete the proof.

By the definition of topologically involutive plane fields, the $C^{1}$ involutive plane fields are dense in $\mathscr{N}$. Let $\widehat{F} \in \mathcal{N}$ be $C^{1}$. Since $\widehat{F}$ is uniquely integrable, $\widehat{F} \in \mathscr{I}_{\rho}$. For $\varepsilon>0$, let $\mathscr{O}_{\varepsilon}(\widehat{F})$ be the neighborhood of $\widehat{F}$ of those $F \in \mathscr{N}$ which, on each $B_{i}$, differ from $\widehat{F}$ in local coordinates by at most $\varepsilon$. That is, for any $U_{i}$, if $\hat{\mathbf{A}}, \mathbf{A} \in C^{0}\left(U_{i}, L\left(\mathbb{R}^{k}, \mathbb{R}^{n-k}\right)\right)$ are defined in local coordinates by $\widehat{F}_{y}=\operatorname{graph}\left(\hat{\mathbf{A}}_{y}\right)$ and $F_{y}=\operatorname{graph}\left(\mathbf{A}_{y}\right)$, then $\left\|\hat{\mathbf{A}}_{y}-\mathbf{A}_{y}\right\|<\varepsilon$ for all $y \in B_{i}$. We want to show $\mathscr{O}_{\varepsilon}(\widehat{F}) \subset \mathscr{I}_{\rho}$ for $\varepsilon$ sufficiently small.

Suppose $F \in \mathscr{O}_{\varepsilon}(\widehat{F})$ and $x \in C_{i}$. Let $N_{1}$ and $N_{2}$ be two integrable manifolds of $F$ through $x$. We want $\delta_{i}\left[N_{1}, N_{2}\right]<\rho$ for $\varepsilon$ small. Let $\hat{\mathbf{A}}$ and $\mathbf{A}$ be as above. Since $\hat{\mathbf{A}}$ is $C^{1}$ (and integrable), there is a $C^{1}$ foliation on $D_{2 d}^{k} \times D_{2 d}^{n-k}$ integrating $\hat{\mathbf{A}}$ whose leaves are all graphs over $D_{2 d}^{k}$. Therefore, there is a $C^{1}$ foliation chart $H: D_{2 d}^{k} \times D_{2 d}^{n-k} \rightarrow \mathbb{R}^{k} \times \mathbb{R}^{n-k}$ taking these leaves to flat planes.

Let $\left(u_{0}, v_{0}\right)$ be the coordinates of $x$. Let $N_{1}$ and $N_{2}$ be the graphs of $g_{1}$ and $g_{2}$. For $j=1,2$, we have $g_{j}\left(u_{0}\right)=v_{0}$, and $\left(D g_{j}\right)_{u}=\mathbf{A}_{\left(u, g_{j} u\right)}$ for all $u \in V_{j}$. Let $\hat{r}$ be defined as above. Fix $u$ with $\left\|u-u_{0}\right\| \leq 1 / 2 \hat{r}$, and for $t \in[0,1]$, define $\mu(t):=u_{0}+t\left(u-u_{0}\right)$. Let $\gamma_{j}(t):=\left(\mu(t), g_{j}(\mu(t))\right), j=1,2$. $\gamma_{j}(t)$ is a curve in the graph of $g_{j}$ from $\left(u_{0}, v_{0}\right)$ to $\left(u, g_{j}(u)\right)$. Since $H$ is a foliation chart, $(D H)_{(u, v)}$ takes the tangent plane at $(u, v)=\operatorname{graph}\left(\hat{\mathbf{A}}_{(u, v)}\right)$ to $\mathbb{R}^{k} \times\{0\}$. Consequently, $(D H)_{(u, v)}\left[\mathbf{I} \hat{\mathbf{A}}_{(u, v)}\right]^{\top}=[\mathbf{I} 0]^{\top}$ for all $(u, v)$, and in particular,

$$
(D H)_{\gamma_{1}(t)}\left[\mathbf{I} \hat{\mathbf{A}}_{\gamma_{1}(t)}\right]^{\top}=(D H)_{\gamma_{2}(t)}\left[\mathbf{I} \hat{\mathbf{A}}_{\gamma_{2}(t)}\right]^{\top} \text {. }
$$

Therefore, we have the following:

$$
\begin{aligned}
& \left\|\left(g_{2}-g_{1}\right)(u)\right\|=\left\|\left(u, g_{2} u\right)-\left(u, g_{1} u\right)\right\| \\
& \leq \operatorname{Lip}\left(H^{-1}\right)\left\|H\left(u, g_{2} u\right)-H\left(u, g_{1} u\right)\right\|, \\
& \left\|H\left(u, g_{2} u\right)-H\left(u, g_{1} u\right)\right\| \\
& \leq \int_{0}^{1}\left\|(D H)_{\gamma_{2}(t)}\left[\mathbf{I} \mathbf{A}_{\gamma_{2}(t)}\right]^{\top}-(D H)_{\gamma_{1}(t)}\left[\begin{array}{ll}
\mathbf{I} & \mathbf{A}_{\gamma_{1}(t)}
\end{array}\right]^{\top}\right\|\left\|u-u_{0}\right\| d t,
\end{aligned}
$$


and

$$
\begin{aligned}
& \left\|(D H)_{\gamma_{2}(t)}\left[\mathbf{I} \mathbf{A}_{\gamma_{2}(t)}\right]^{\top}-(D H)_{\gamma_{1}(t)}\left[\mathbf{I} \mathbf{A}_{\gamma_{1}(t)}\right]^{\top}\right\| \\
& \quad \leq\left\|(D H)_{\gamma_{2}(t)}\left[\mathbf{I} \mathbf{A}_{\gamma_{2}(t)}\right]^{\top}-(D H)_{\gamma_{2}(t)}\left[\mathbf{I} \hat{\mathbf{A}}_{\gamma_{2}(t)}\right]^{\top}\right\| \\
& \quad+\left\|(D H)_{\gamma_{1}(t)}\left[\mathbf{I} \hat{\mathbf{A}}_{\gamma_{1}(t)}\right]^{\top}-(D H)_{\gamma_{1}(t)}\left[\mathbf{I} \mathbf{A}_{\gamma_{1}(t)}\right]^{\top}\right\| \\
& \quad \leq\left\|(D H)_{\gamma_{2}(t)}\right\|\left\|\mathbf{A}_{\gamma_{2}(t)}-\hat{\mathbf{A}}_{\gamma_{2}(t)}\right\|+\left\|(D H)_{\gamma_{1}(t)}\right\|\left\|\mathbf{A}_{\gamma_{1}(t)}-\hat{\mathbf{A}}_{\gamma_{1}(t)}\right\| \\
& \quad \leq 2 \operatorname{Lip}(H) \sup \left\{\left\|\mathbf{A}_{(u, v)}-\hat{\mathbf{A}}_{(u, v)}\right\|:\|u\|,\|v\| \leq 2 d\right\}<2 \varepsilon \operatorname{Lip}(H) .
\end{aligned}
$$

Consequently, $\left\|\left(g_{2}-g_{1}\right)(u)\right\|<2 \varepsilon \operatorname{Lip}(H) \operatorname{Lip}\left(H^{-1}\right)\left\|u-u_{0}\right\| \leq \rho\left\|u-u_{0}\right\|$ for $\varepsilon$ sufficiently small. Since $H$ depended only on $\widehat{F}$ and the fixed local coordinates on $U_{i}$, we can choose one $\varepsilon$ so that the above inequalities hold in each $U_{i}$. Hence, $\mathscr{O}_{\varepsilon}(\widehat{F}) \subset \mathscr{I}_{\rho}$.

Since the set of $C^{1} F$ is dense in $\mathscr{I}_{\rho}$, this implies $\mathscr{I}_{\rho}$ contains an open and dense set. Consequently, $\mathscr{I}(K)$ is residual, which completes the proof.

\section{REFERENCES}

1. R. Abraham, J. Marsden, and T. Tatiu, Manifolds, tensor analysis, and applications, Addison-Wesley, Reading, MA, 1983.

2. G. Choquet, Lectures on analysis, Vol. 1, W. A. Benjamin, New York, 1969.

3. M. Hirsch, Differential topology, Graduate Texts in Math., vol. 33, Springer-Verlag, New York, 1976.

4. W. Orlicz, Zue theorie der differentialgleichung $y^{\prime}=f(x, y)$, Bull. Int. Acad. Polon. Sci. A 8/9 (1932), 221-228.

Department of Mathematical Sciences, The University of Cincinnati, Cincinnati, OHIO 45221-0025

E-mail address: pat.mcswiggen@uc.edu 